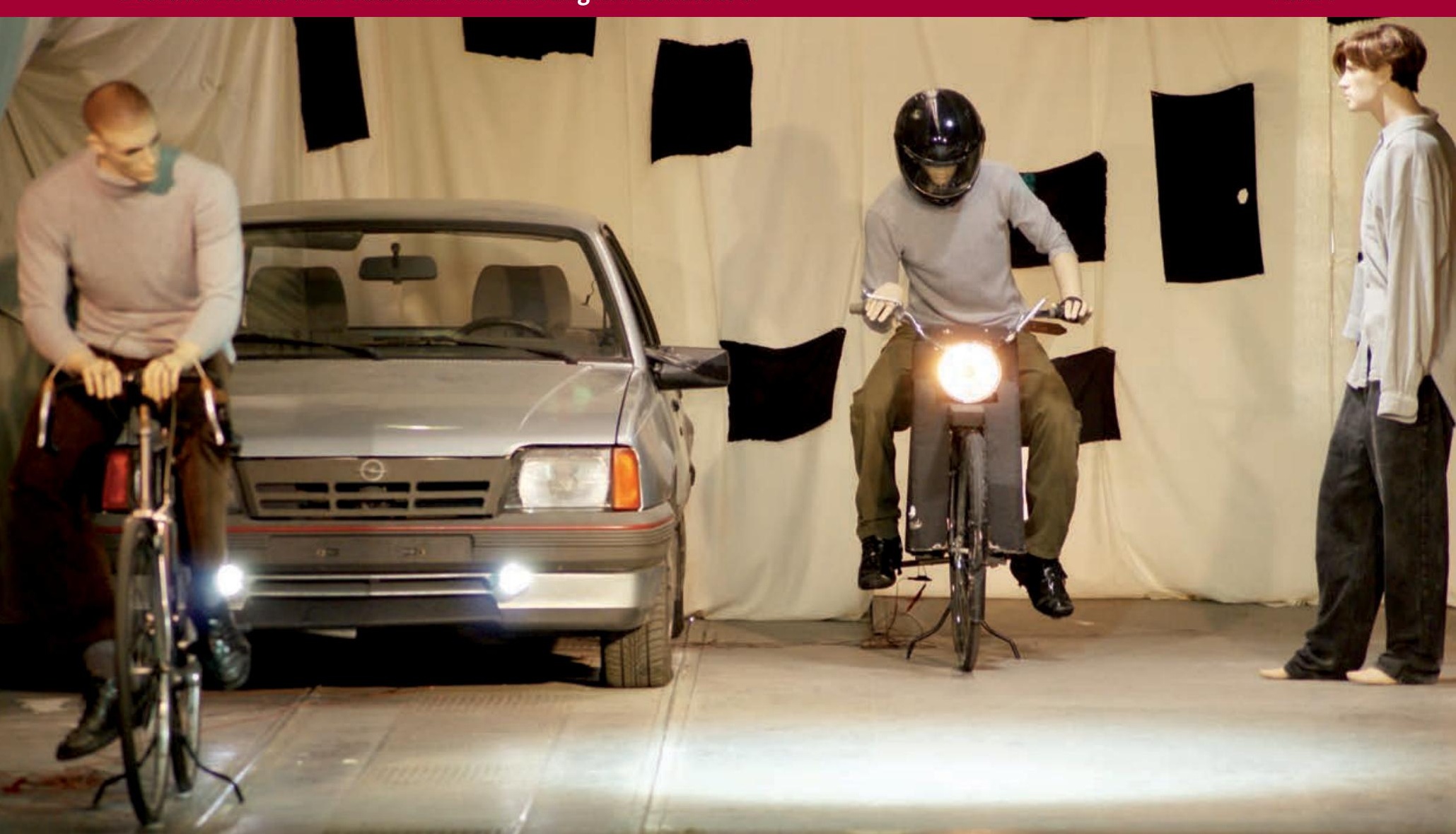

Unfallforschung kompakt

\title{
Tagfahrlicht an Pkw - Analyse des Einflusses auf die Verkehrssicherheit
}

Unfallforschung der Versicherer 
Impressum

Gesamtverband der Deutschen Versicherungswirtschaft e. V.

Unfallforschung der Versicherer

Wilhelmstraße 43/43G, 10117 Berlin

Postfach 0802 64, 10002 Berlin

unfallforschung@gdv.de

www.udv.de

Redaktion: Dr. Matthias Kühn

Layout: Franziska Gerson Pereira

Bildnachweis: UDV und Quellenangaben

Erschienen: 04/2011 


\section{Vorbemerkung}

Vom 07.02.2011 an dürfen in Europa nur noch neue Fahrzeugtypen zugelassen werden, die mit Tagfahrlicht ausgestattet sind. Tagfahrleuchten sollen als energiesparende und langlebige Alternative zum Abblendlicht die Wahrnehmbarkeit von Fahrzeugen verbessern, und somit aktiv zur Verkehrssicherheit beitragen. Die Befürworter der Maßnahme berufen sich auf Studien, die erhebliche Unfallreduktionen prognostizieren. Die konservativeren Schätzungen bewegen sich zwischen 3 und 4\% [1, 2]. Hingegen fürchten die Tagfahrlichtgegner um die Sicherheit schwächerer Verkehrsteilnehmer. 


\section{Inhalt}

Vorbemerkung 2

1 Ziel 4

2 Die Forschungslandschaft im Überblick 4

3 Fahrstudie mit Blickbewegungsmessung 5

$4 \quad$ Laborstudie im Lichtkanal 6

$\begin{array}{lll}5 & \text { Fahrsimulatorstudie } & 7\end{array}$

$\begin{array}{lll}6 & \text { Fazit } & 8\end{array}$

$\begin{array}{ll}\text { Literatur } & 9\end{array}$ 


\section{Ziel}

Im Auftrag der Unfallforschung der Versicherer (UDV) hat die Human-Factors-Consult $\mathrm{GmbH}$ in Kooperation mit dem Fachgebiet Lichttechnik der TU Berlin die Sicherheitsmaßnahme Tagfahrlicht auf Sicherheits- und Negativeffekte geprüft. Ziel des Projekts war die empirische Prüfung folgender Fragestellungen, um auf Basis der Untersuchungsergebnisse Aussagen zur Unfallentwicklung für Pkw und schwächere Verkehrsteilnehmer ableiten zu können:

- Aussagen zu Sicherheitsvorteilen und Risiken: Welchen Effekt zeigt Licht an Pkw für PkwFahrer und schwächere Verkehrsteilnehmer?

- Vergleich ausgewählter Unfalltypen: Gibt es Effektunterschiede?

- Beurteilung der Nachhaltigkeit der Effekte: Nivellieren sich Effekte in Folge der Gewöhnung an Licht an Pkw?

- Vergleich von Abblendlicht und Tagfahrlicht: Wo liegt der Unterschied?

- Variation der Lichtbedingungen: Beeinflussen unterschiedliche Kontrastverhältnisse die Effekte?

- Kontrolle demografischer und situativer Merkmale:

Was moderiert die Wirkung von Licht an Pkw?

Innerhalb einer Projektlaufzeit von zweieinhalb Jahren wurden drei empirische Studien durchgeführt:

(1) Fahrstudie mit Blickbewegungsmessung in Dänemark und Deutschland

(2) Laborstudie im Lichtkanal der TU Berlin

(3) Fahrsimulatorstudie mit Erfassung des Blick- und Fahrverhaltens.

Die Kombination verschiedener empirischer Methoden ermöglichte es, unterschiedliche Facetten des Themas vertiefend zu prüfen, die Aussagen gegenseitig zu validieren, und die Vorteile experimenteller Kontrolle mit Aussagen zur Übertragbarkeit der Ergebnisse in den realen Straßenverkehr zu verbinden.

\section{Die Forschungslandschaft im Überblick}

Zu Projektbeginn erfolgte eine umfassende Sichtung und Bewertung der Literatur nach ausgewählten Kriterien zur Beurteilung der methodischen Güte und Aussagekraft. Dies ermöglichte eine Unterscheidung glaubwürdig belegter Effekte von empirisch nicht (ausreichend) gesicherten oder fehlenden Aussagen.

Die Mehrzahl der Studien basiert auf Unfallstatistiken für Fahrzeugmodelle mit vs. ohne Tagfahrlicht (bzw. eingeschaltetes Abblendlicht) oder auf nationalen Unfalldaten vor vs. nach Einführung der Lichtpflicht am Tag.

Die berichteten Unfallrückgänge liegen oft im zweistelligen Bereich und sind zumeist nicht signifikant, d.h. sie liegen im Rahmen statistischer Zufallsschwankungen. Deren unzulässige Interpretation als Sicherheitseffekte führt mitunter zu prognostizierten Unfallreduktionen von bis zu $25 \%$ [3], während der Effekt weder als Sicherheitsgewinn noch als Risikofaktor plausibel belegt ist. Bei Studien, die Unfallzahlen als Maß heranziehen, ist der Effekt entweder nicht von anderen Einflussfaktoren zu trennen (z. B. nationale Studien, Flottenstudien) oder es fehlen hinreichend große Fallzahlen (Experimentaldesign), die für eine teststarke statistische Analyse erforderlich wären.

Im Labor zeigt sich eine verbesserte Erkennbarkeit beleuchteter Pkw, während bislang kein Nachteil für schwächere Verkehrsteilnehmer nachweisbar war. Aus verschiedenen Gründen ist die Übertragbarkeit dieser Ergebnisse auf 
den realen Straßenverkehr nicht gegeben. Eine Fahrstudie [4] interpretiert geringfügig längere Blickzuwendungen auf beleuchtete Pkw als Blickbindung und schließt daraus auf eine Gefährdung für schwächere Verkehrsteilnehmer, ohne diese Folgerung empirisch zu stützen.

Überraschend ist die mitunter ausgeprägte Positionierung pro vs. contra Tagfahrlicht, zumal die Vor- und Nachteile von Tagfahrlicht wie seine Wirkmechanismen (Aufmerksamkeitslenkung, Blickbindung) trotz umfassender Forschungsarbeiten noch nicht schlüssig belegt sind.

\section{Fahrstudie mit Blickbewegungsmessung}

Im ersten Teil der Fahrstudie wird das natürliche Blickverhalten von Pkw-Fahrern auf beleuchtete und unbeleuchtete Pkw und auf schwächere Verkehrsteilnehmer untersucht. Der Fokus liegt auf Knotenpunkten im innerstädtischen Straßenverkehr, um Effekte für ausgewählte Unfalltypen (Abbiegen und Einbiegen/Kreuzen) zu vergleichen. Im zweiten Teil wird geprüft, ob die Wahrnehmung von
Motorradfahrern durch Licht an Pkw beeinträchtigt wird.

\section{Teil 1: Natürliches Blickverhalten auf Pkw mit vs. ohne Licht}

Die Aufgabe der Probanden lautete, eine vorgegebene Versuchsstrecke in Deutschland und Dänemark abzufahren. Erfasst wurden die Blickzuwendungen von 50 Probanden auf Pkw mit und ohne Licht am Tag, Fußgänger, Fahrradfahrer und Motorradfahrer. Situative Bedingungen und soziodemografische Einflussfaktoren wurden mit erhoben und geprüft. Die der Auswertung zugrunde liegende Datenbasis umfasst die Blickzuwendungen auf 2.500 Verkehrsteilnehmer.

\section{Fragestellung}

- Gibt es Unterschiede in den Blickzuwendungen auf Pkw mit vs. ohne Licht?

- Beeinflusst Licht an Pkw das Blickverhalten gegenüber schwächeren Verkehrsteilnehmern?

- Welches Ergebnismuster zeigt eine Differenzierung der Ergebnisse nach Unfalltyp?

- Welche Rolle spielen situative Faktoren wie z. B. Umgebungshelligkeit?

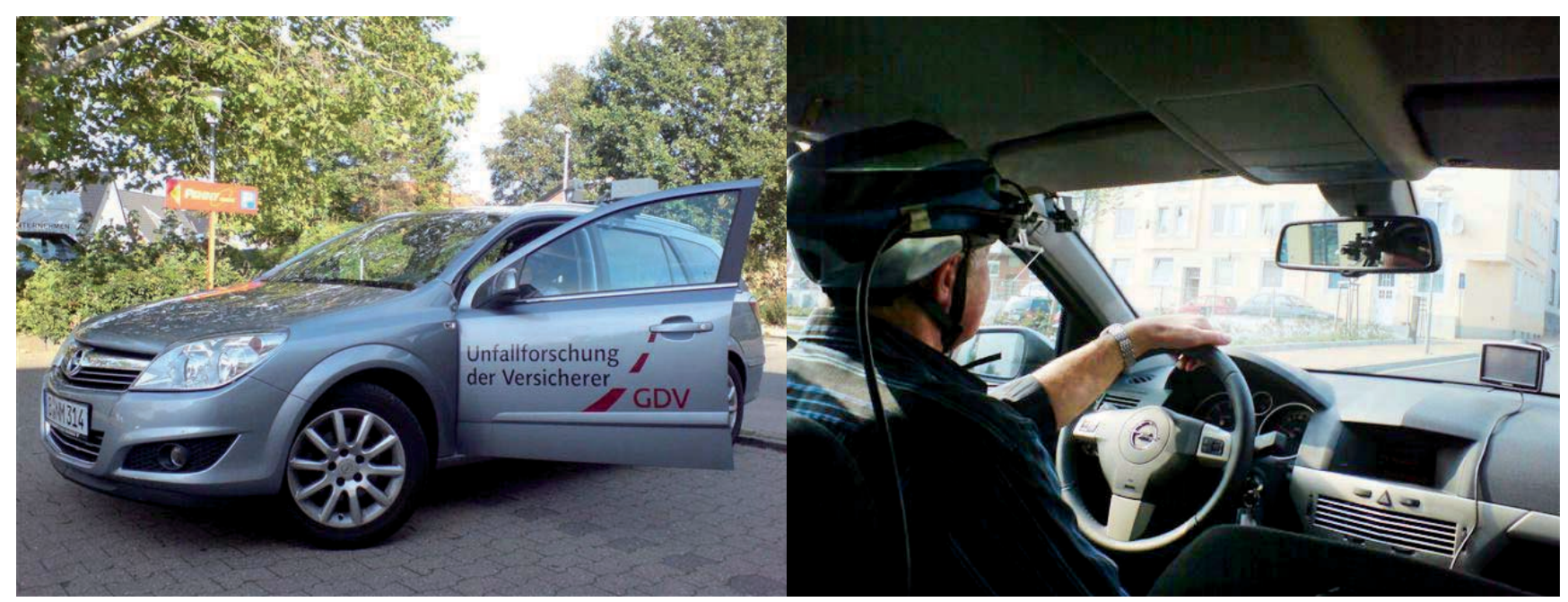

Abbildung 1: Versuchsfahrzeug der Fahrstudie (links) und Proband mit Blickbewegungsmesssystem (rechts) 
- Gibt es Gewöhnungseffekte für Licht an Pkw, prüfbar durch den Vergleich von Probanden aus Dänemark und Deutschland?

\section{Ergebnisse}

Das Blickverhalten von Autofahrern wird durch Licht an Pkw nur geringfügig beeinflusst. Die Blickerstzuwendung, die mittlere Blickdauer, die Anzahl an Blicken und die kumulierte Blickdauer zeigen keinen signifikanten Effekt für Licht an Pkw bei Sonne. Bei Bewölkung oder Regen ist die kumulierte Blickdauer für Pkw mit Licht um 0,24 s höher. Dies impliziert eine höhere Salienz von Pkw mit Licht. Dieser Unterschied wird aber als praktisch nicht bedeutsam für die Verkehrssicherheit eingeschätzt. Unterschiede im Blickverhalten gegenüber schwächeren Verkehrsteilnehmern bei Pkw mit Licht konnten nicht nachgewiesen werden. Es gab keine Unterschiede im Blickverhalten zwischen Dänen und Deutschen, die auf Licht an Pkw zurückzuführen sind. Ein statistisch tragfähiger Vergleich der Unfalltypen war in Ermangelung der Fallzahlen nicht möglich. Ersatzweise erfolgte eine Analyse nach der Richtung, aus der Fahrzeuge am Knotenpunkt sichtbar werden. Ein moderierender Einfluss war nicht feststellbar.

Teil 2: Wahrnehmung motorisierter Zweiräder

In der zweiten Hälfte der Testfahrt waren die Probanden instruiert, beim Abfahren einer vorgegebenen Versuchsstrecke alle motorisierten Zweiräder anzusagen, die ihnen im Straßenverkehr auffallen, ohne jedoch den Verkehr gezielt danach abzusuchen.

\section{Fragestellung}

- Werden motorisierte Zweiräder bei Licht an Pkw häufiger übersehen?

- Moderieren situative Bedingungen, wie z.B. die Umgebungshelligkeit, die Ergebnisse?

\section{Ergebnisse}

Der Vergleich von wahrgenommenen mit übersehenen motorisierten Zweirädern zeigt keinen signifikanten Effekt von Licht an Pkw. Auch wenn einige situative, personen- und objektbezogene Faktoren die Wahrnehmung motorisierter Zweiräder im Straßenverkehr begünstigen oder beeinträchtigen, führt keiner in Verbindung mit Licht an Pkw zu einem signifikanten Ergebnis.

\section{Laborstudie im Lichtkanal}

Bei einer sehr kurzen Darbietung (120ms) einfacher Verkehrsszenen sollten die Probanden während der Bearbeitung einer kontinuierlichen Nebenaufgabe über die An- bzw. Abwesenheit eines schwächeren Verkehrsteilnehmers entscheiden und diesen identifizieren. Die Szenerie wurde unter zwei Lichtbedingungen, taghell vs. Dämmerung, dargeboten. Teil der Szenerie war ein Pkw mit variierter Lichtkonfiguration: kein Licht vs. Abblendlicht (Halogen) vs. Tagfahrlicht (LED) und entweder

- Kein weiterer Verkehrsteilnehmer

- Fahrradfahrer (vor Pkw)

- Fußgänger (weit neben Pkw)

- Motorradfahrer (vor vs. neben Pkw).

\section{Fragestellung}

- Welchen Einfluss zeigt die Pkw-Beleuchtung auf die Erkennungsrate, also die Wahrnehmbarkeit und Identifikation schwächerer Verkehrsteilnehmer?

- Ändert sich mit der Pkw-Beleuchtung die Reaktionszeit, also die Zeitspanne zwischen Darbietung der Szenerie und Entscheidung über die Anwesenheit eines Verkehrsteilnehmers?

- Wird die Sicherheit des Urteils durch die PkwBeleuchtung beeinflusst? 

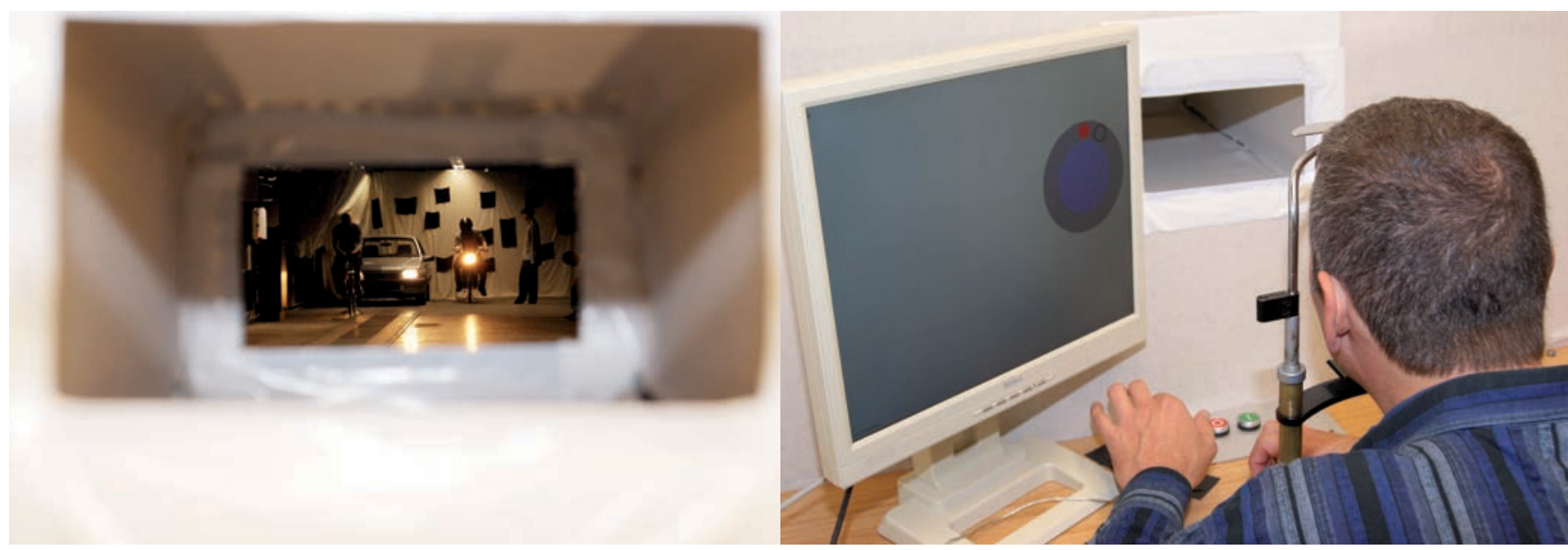

Abbildung 2: Sicht des Probanden auf die Szenerie im Lichtkanal (links) und Position des Probanden mit Nebenaufgabe (rechts)

\section{Ergebnisse}

Für die Erkennungsrate zeigt sich kein Einfluss der Pkw-Beleuchtung. Schwächere Verkehrsteilnehmer werden bei Licht an Pkw nicht häufiger übersehen. Bei den Reaktionszeiten zeichnet sich nur für eine von vier Situationen, dem neben dem Pkw platzierten Motorrad, eine signifikante Erhöhung um 0,2 s bei Abblendlicht und Tagfahrlicht gegenüber der Bedingung ohne Licht $a b$. Hier verringert sich auch die Sicherheit des eigenen Urteils. Die längere Reaktionszeit stellt allerdings nur einen Angleich an die längeren Reaktionszeiten dar, die man bei den anderen Verkehrsteilnehmern beobachtet hat. Damit finden sich auch im Laborexperiment keine hinreichenden Belege, die auf eine erhöhte Gefährdung schwächerer Verkehrsteilnehmer durch Licht an Pkw schließen lassen.

\section{Fahrsimulatorstudie}

Für die Versuche wurde der Fahrsimulator der Professur Verkehrspsychologie an der TU Dresden benutzt. 20 Probanden fuhren eine simulierte Versuchsstrecke ab. Erfasst wurden neben dem Blickverhalten für Motorradfahrer, Fahrradfahrer, Fußgänger und Pkw mit vs. ohne Licht auch das Fahrverhalten (Gap Accep- tance) an innerstädtischen Knotenpunkten. Die Datenbasis umfasst das Fahrverhalten an über 400 Knotenpunkten und die Blickzuwendungen auf 1300 Verkehrsteilnehmer.

\section{Fragestellung}

- Beeinflusst Licht an Pkw das Blickverhalten gegenüber Pkw und schwächeren Verkehrsteilnehmern?

- Erfolgt eine frühere Blickzuwendung auf Pkw mit Licht bzw. eine spätere auf Motorradfahrer, Fahrradfahrer und Fußgänger?

- Welches Ergebnismuster zeigt eine Differenzierung der Ergebnisse nach Unfalltyp?

- Welchen Einfluss hat Licht an Pkw auf die Akzeptanz von Zeitlücken (Gap Acceptance) und den Abstand zu sich nähernden Fahrzeugen an belebten Knotenpunkten?

\section{Ergebnisse}

Es zeigt sich kein signifikanter Einfluss von Licht an Pkw auf das Blickverhalten der Probanden. Zeitpunkt der Blickerstzuwendung, Blickanzahl und -dauer sind für Pkw mit und ohne Licht und für schwächere Verkehrsteilnehmer vergleichbar. Gleiches gilt für die Akzeptanz von Zeitlücken und die Größe des Abstandes zu den sich nähernden Fahrzeu- 


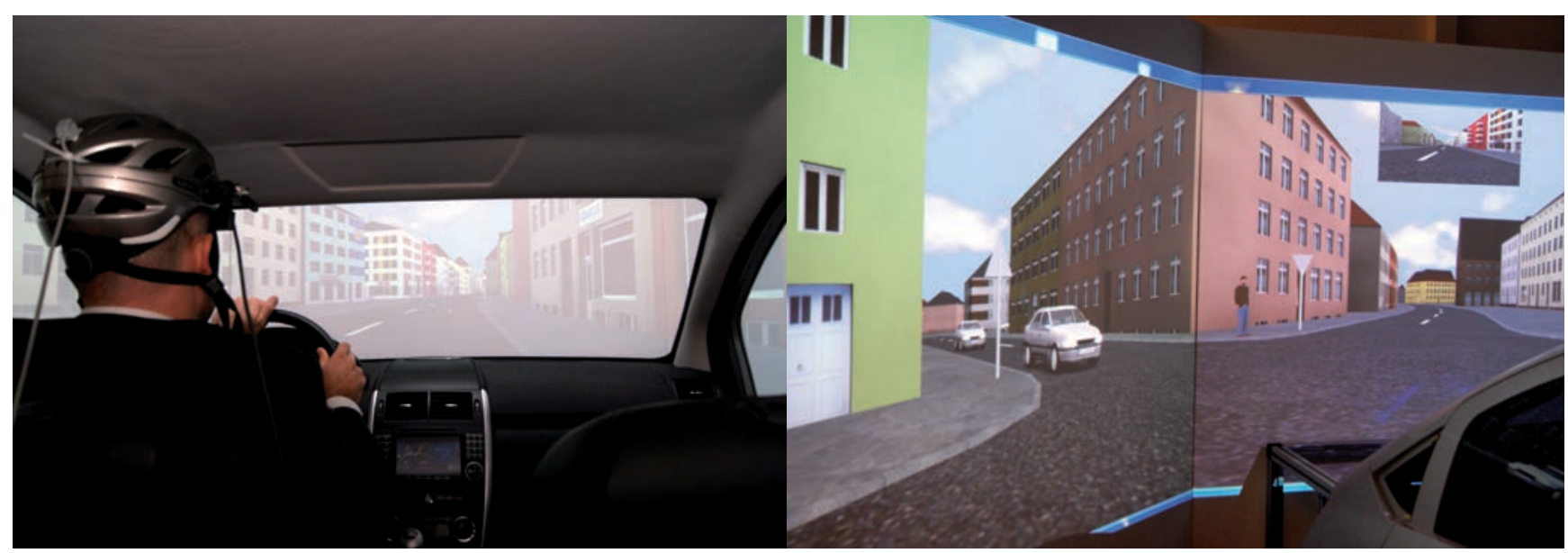

Abbildung 3: Proband im Fahrsimulator mit Blickbewegungsmesssystem (links) und innerstädtische Szenerie (rechts)

gen beim Abbiegen, Einbiegen und Kreuzen. Auch die Analyse des Sonderfalls,Pkw folgt Motorrad', der von Gegnern des Tagfahrlichts als höchstkritisches Szenario angeführt wird, lässt keine Nachteile für Motorräder bei Pkw mit Licht erkennen. Die verschiedenen Unfalltypen weisen keine signifikanten Unterschiede in Blick- und Fahrverhalten auf, die sich auf Licht an Pkw zurückführen lassen.

\section{Fazit}

Es zeigen sich nur geringe Effekte für Licht an Pkw. Bei Bewölkung/Regen ist die kumulierte Blickdauer auf Pkw mit Licht um 0,24 s länger. Die längeren Blickzuwendungen gehen zurück auf häufigere und nicht auf längere Einzelblickzuwendungen und werden deshalb als nicht verkehrssicherheitsrelevant angesehen. Bei Sonne zeigt sich dieser Effekt nicht. Eine frühere oder spätere Blickzuwendung auf beleuchtete Pkw bzw. schwächere Verkehrsteilnehmer ist ebenso wenig feststellbar wie ein Einfluss auf das Fahrverhalten. Die Analyse des Blickverhaltens von Pkw-Fahrern auf Fahrradfahrer und Fußgänger weist ebenso wie der Vergleich übersehener motorisierter Zweiräder über Situationen mit beleuchteten und unbeleuchteten Pkw keinen Effekt für
Licht an Pkw auf. Der im Labor aufgezeigte Einzelbefund einer um 0,2s längere Reaktionszeit bei Darbietung des Motorrads neben einem Pkw mit Licht wird für unbedenklich gehalten. Aus verschiedenen Gründen lässt sie sich am ehesten als methodisches Artefakt des gewählten statischen Versuchsaufbaus erklären und besitzt daher keine praktische Relevanz für dynamische Realfahrten. Die Wirkungen von moderierenden Einflussfaktoren (z. B. Gewöhnungseffekte, Differenzierung nach Unfalltypen, etc.) können nicht abschließend bewertet werden, da der generelle Effekt des Tagfahrlichts zu klein war, trotz einer außergewöhnlich umfangreichen Datenbasis. Selbst kleine Effekte des Tagfahrlichts hätten aufgezeigt werden können - sofern sie denn vorhanden sind. Im Rahmen dieser Studie konnten keine positiven Effekte auf die Verkehrssicherheit durch Tagfahrlicht am Pkw festgestellt werden. Allerdings konnten auch keine negativen sicherheitsrelevanten Einflüsse auf schwächere Verkehrsteilnehmer festgestellt werden. Damit ist kein spürbarer Einfluss von Licht an Pkw auf das Unfallgeschehen zu erwarten.

\section{Weitere Informationen unter: www.udv.de www.youtube.com/unfallforschung}




\section{Literatur}

[1] Schönebeck, S., Ellmers, U., Gail, J., Krautscheid, R. \& Tews, R. (2005). Abschätzung möglicher Auswirkungen von Fahren mit Licht am Tag (Tagfahrleuchten/Abblendlicht) in Deutschland, Bundesanstalt für Straßenwesen.

[2] Elvik, R., Christensen, P. \& Olsen, S. F. (2003). Daytime running lights: A systematic review of effects on road safety, Transportøkonomisk institutt. IR 2.

[3] Koornstra, M., Bijleveld, F. \& Hagenzieker, M. (1997). The Safety Effects of Daytime Running Lights: A perspective on Daytime Running Lights (DRL) in the EU: the statistical reanalysis and a meta-analysis of 24 independent DRL evaluations as well as an investigation of possible policies on a DRL regulation in the EU, SWOV Institute for Road Safety Research.

[4] Pfleger, E. (2007). Untersuchung von blicktechnischen Interaktionen im realen Straßenverkehr in Ortsgebieten und Freiland: Blickanalysen mit dem Viewpointsystem unter Einsatz modernster High-Tech-Rasteranalyseprogramme zur wissenschaftlichen Bewertung der Auswirkungen von Licht am Tag (Positionspapier Erstinformation), EPIGUS - Institut für ganzheitliche Unfall- und Sicherheitsforschung. 


\section{GDV}

DIE DEUTSCHEN VERSICHERER

\section{Gesamtverband der Deutschen Versicherungswirtschaft e.V.}

Wilhelmstraße 43/43G, 10117 Berlin

Postfach 0802 64, 10002 Berlin

Tel.: 030/20 20 - 50 00, Fax: 030/2020-6000

www.gdv.de, www.udv.de 